\title{
MicroRNA-21, induced by high glucose, modulates macrophage apoptosis via programmed cell death 4
}

\author{
YUAN-YUAN SHANG ${ }^{1}$, NING-NING FANG ${ }^{1,2}$, FENG WANG $^{1}$, HUI WANG ${ }^{1}$, ZHI-HAO WANG ${ }^{1}$, \\ MENG-XIONG TANG ${ }^{1}$, JIE PENG ${ }^{1}$, YUN ZHANG ${ }^{1}$, WEI ZHANG ${ }^{1}$ and MING ZHONG ${ }^{1}$ \\ ${ }^{1}$ Key Laboratory of Cardiovascular Remodeling and Function Research Chinese Ministry of Education and \\ Chinese Ministry of Public Health, Department of Cardiology, Qilu Hospital of Shandong University, Ji'nan, Shandong 250012; \\ ${ }^{2}$ Key Laboratory of Cardiovascular Remodeling and Function Research Chinese Ministry of Education and \\ Chinese Ministry of Public Health, Department of Anesthesiology, Qilu Hospital of Shandong University, \\ Ji'nan, Shandong 250033, P.R. China
}

Received May 10, 2014; Accepted January 27, 2015

DOI: $10.3892 / \mathrm{mmr} .2015 .3398$

\begin{abstract}
MicroRNA-21 (miR-21) has been found to promote cell proliferation and survival. It has also been shown to exhibit an increased expression in a number of forms of cardiovascular disease. However, the mechanisms underlying the involvement of miR-21 in atherosclerosis remain to be elucidated. In the present study, it was demonstrated that miR-21 was upregulated in a time-dependent manner in response to high-concentration glucose stimulation in Raw 264.7 macrophages. High concentrations of glucose induce macrophage apoptosis. miR-21-inhibited macrophages treated with a normal concentration of glucose exhibited increased levels of cell apoptosis and augmented levels of activated caspase-3, while cells treated with an miR-21 inhibitor and a high concentration of glucose, revealed significantly increased levels of apoptosis. In addition, inhibition of miR-21 increased mRNA and protein levels of programmed cell death 4 (PDCD4), which, by contrast, were reduced in miR-21-inhibited cells that had been treated with a high concentration of glucose. In conclusion, miR-21 is sensitive to high-concentration glucose treatment in macrophages, and appears to have a protective effect in macrophage apoptosis induced by high concentrations of glucose via PDCD4.
\end{abstract}

Correspondence to: Professor Ming Zhong, Key Laboratory of Cardiovascular Remodeling and Function Research Chinese Ministry of Education and Chinese Ministry of Public Health, Department of Cardiology, Qilu Hospital of Shandong University, 107 Wen Hua Xi road, Ji'nan, Shandong 250012, P.R. China E-mail: zhongmingzm@gmail.com

Key words: microRNA-21, programmed cell death 4, macrophage, apoptosis, high glucose, atherosclerosis

\section{Introduction}

Cardiovascular disease is the leading cause of mortality among individuals with diabetes and diabetes is a significant risk factor for the development of cardiovascular disease $(1,2)$. Patients with diabetes have a clear predilection for atherosclerosis, and $>80 \%$ diabetic patients succumb to acute coronary syndrome $(1,2)$. Apoptosis of macrophages contributes significantly to the development of vulnerable atherosclerotic plaques (3-5). As demonstrated in early postmortem studies, apoptosis of macrophages is increased in atherosclerotic plaques in patients with diabetes (6). The signal transduction of apoptosis in macrophages involves a complex network, and the mechanism underlying macrophage apoptosis remains to be elucidated.

MicroRNAs (miRNAs) are small, endogenous, non-coding RNAs of $\sim 22$ nucleotides that have a broad impact on gene expression through translational repression or post-transcriptional suppression $(7,8)$. It is estimated that there are $>700$ miRNAs in humans and that the number of miRNA targeted genes in the human genome is as high as $1,000(9,10)$. A single miRNA may regulate the expression of multiple target genes. Therefore, miRNAs may regulate various biological processes, including development, differentiation, carcinogenesis, cell proliferation and apoptosis (11). To date, miRNAs have been shown to have crucial roles in a number of diseases, including cancer, infectious diseases, diabetes and cardiovascular disease (12-15).

Recently, microRNA-21 (miR-21), which has been reported to be involved in a number of forms of cardiovascular disease has become an increasing focus in research. miR-21, which is considered to be an oncomiR (16), is expressed in diverse types of cardiovascular cells, including cardiomyocytes, cardiac fibroblasts, vascular smooth muscle cells and human umbilical vein endothelial cells (HUVECs) (17-20). In addition, altered expression of miR-21 may lead to the development of cardiovascular disease (19). miR-21 has been reported to be a novel regulator for neointimal lesion formation (21). In the rat carotid artery, following angioplasty, miR-21 was found to be aberrantly expressed, and downregulation of this 
overexpression of miR-21, decreased neointima formation. The expression of miR-21 has been found to be significantly upregulated in atherosclerotic arteries (22). However, the mechanisms underlying miR-21 involvement in atherosclerosis remain to be elucidated. miR-21 has been observed to target and downregulate programmed cell death 4 (PDCD4) (23) and to promote cell proliferation. In addition, overexpression of miR-21 represses normal apoptotic signaling, and inhibition of miR-21 increases cell apoptosis $(23,24)$. Therefore, cell apoptosis regulated by miR-21 may be a potential mechanism contributing to the development of atherosclerosis and miR-21 may be involved in macrophage apoptosis.

It was hypothesized that miR-21 is able to resist macrophage apoptosis induced by high glucose concentrations via PDCD4. In the present study, in order to investigate the effect of miR-21 on macrophage apoptosis, Raw 264.7 macrophages were stimulated with high glucose concentrations and transfected with an anti-miR-21 inhibitor. The levels of miR-21 expression, PDCD4 expression and cell apoptosis were subsequently measured.

\section{Materials and methods}

Cell culture and treatment. The Raw 264.7 murine monocytic cell line was routinely maintained in Dulbecco's modified Eagle's medium (5,5 mM D-glucose) supplemented with $10 \%$ fetal bovine serum and cultured at $37^{\circ} \mathrm{C}$ in a humidified atmosphere containing $5 \% \mathrm{CO}_{2}$. Cells were passaged every 2-3 days in order to maintain exponential growth. Treatment of Raw 264.7 macrophages with $5.5 \mathrm{mM}$ glucose (normal glucose, $\mathrm{NG}$ ), $25 \mathrm{mM}$ glucose (high glucose, $\mathrm{HG}$ ), or $25 \mathrm{mM}$ mannose (osmotic control, OC) were performed in serum-free media.

Reverse transcription quantitative polymerase chain reaction (RT-qPCR) analysis of miR-21 and PDCD4 mRNA expression. Total RNA was extracted from treated cells using the mirVana miRNA isolation kit (Ambion, Inc., Austin, TX, USA) or TRIzol (Invitrogen Life Technologies, Carlsbad, CA, USA). For analysis of the expression of miR-21, $10 \mathrm{ng}$ total RNA was reverse transcribed into cDNA using MultiScribe reverse transcriptase (Applied Biosystems, Foster City, CA, USA). qPCR was performed using a standard TaqMan PCR protocol according to manufacturer's instructions (Applied Biosystems). The relative miR-21 expression level was then calculated using the $2^{-\triangle \Delta C T}$ method (25) and normalized to the expression of U6. Relative levels of PDCD4 mRNA were examined using SYBR green quantitative reverse transcription-PCR (Applied Biosystems) and normalized to $\beta$-actin. The specific primer sequences were as follows: Forward: 5'-GGTGGATGTGAAAGATCCAAA-3' and reverse: 5'-CATCCAGGGGCAAAACTACA-3' for PDCD4 and forward: 5'-CAACTTGATGTATGAAGGCTTTGGT-3' and reverse: 5'-ACTTTTATTGGTCTCAAGTCAGTGTACAG-3' for $\beta$-actin.

Oligonucleotide transfection. For miR-21 inhibition, cells were plated in 12-well plates at $3 \times 10^{5}$ cells $/ \mathrm{ml}$ and incubated at $37^{\circ} \mathrm{C}$ in a $5 \% \mathrm{CO}_{2}$ atmosphere. Cells at $50-70 \%$ confluence were transfected with $60 \mathrm{nM}$ of anti-miR-21 inhibitor via use of Lipofectamine 2000 reagent (Invitrogen Life Technologies).
Cells were incubated for $24 \mathrm{~h}$ post-transfection prior to treatment with NG or HG. The anti-miR ${ }^{\mathrm{TM}}$ miRNA inhibitor (cat no. P/N: AM17000) and the negative control (cat no. P/N: AM17010) were purchased from Applied Biosystems.

Western blot analysis. Total protein from cells was extracted using a radioimmunoprecipitation assay buffer $(1 \mathrm{mM} \mathrm{MgCl}$; $10 \mathrm{mM}$ Tris-HCl, pH 7.4; $1 \%$ Triton X-100; 0.1\% SDS; and 1\% NP-40). Proteins were separated on NuPAGE 4-12\% Bis-Tris gels (Invitrogen Life Technologies), transferred to nitrocellulose membranes (Merck Millipore Ltd., Carrigtwohill, Ireland) and incubated with a rabbit polyclonal anti-PDCD4 antibody (sc-292504; 1:1,000; Santa Cruz Biotechnology, Santa Cruz, CA, USA), rabbit polyclonal anti-caspase-3 antibody (sc-7148; 1:200; Santa Cruz Biotechnology) or a rabbit polyclonal $\beta$-actin antibody (ab8227; 1:1,000; Abcam, Cambridge, $\mathrm{UK}$ ), followed by rabbit immunoglobulin $\mathrm{G}$ horseradish peroxidase-conjugated secondary antibody (ab6721; 1:1,000; Abcam). Blots were developed using Supersignal West Dura extended duration substrate (Perbio, Cheshire, UK). Images were captured using a Chemigenius imaging system (Syngene, Cambridge, UK).

Flow cytometry analysis of cell apoptosis using propidium iodide-Annexin V staining. Cells plated in 12-well plates were transfected with anti-miR-21 inhibitor or negative control for $24 \mathrm{~h}$, and then treated with NG or HG for $48 \mathrm{~h}$. The apoptotic ratio was analyzed using an Annexin $\mathrm{V}$ fluorescein isothiocyanate apoptosis detection kit (Bipec Biopharma Corporation, Cambridge, MA, USA) on a BD FACSCalibur ${ }^{\mathrm{TM}}$ system (Becton-Dickinson, San Jose, CA, USA) according to the manufacturer's instructions. The experiments were repeated three times.

Statistical analysis. Data are presented as the mean \pm standard error of the mean. Comparisons among groups were assessed using a one-way analysis of variance. Analysis was conducted using SPSS 16.0 (SPSS, Inc., Chicago, IL, USA). $\mathrm{P}<0.05$ was considered to indicate a statistically significant difference.

\section{Results}

miR-21 expression is upregulated by high levels of glucose. In order to examine whether the expression of miR-21 in macrophages was regulated by high concentrations of glucose, Raw 264.7 macrophages were treated with $5.5 \mathrm{mM}$ glucose (NG), $25 \mathrm{mM}$ glucose (HG) or $25 \mathrm{mM}$ mannose (OC) for various time periods $(0,1,3,6,12$ and $24 \mathrm{~h})$. TaqMan RT-qPCR demonstrated that the expression of miR-21 mRNA was significantly upregulated by $\mathrm{HG}$, whereas it was reduced by OC (Fig. 1). Compared with the NG group, a non-significant increasing trend was observed in the $\mathrm{HG}$ group. The increase in miR-21 expression at $6 \mathrm{~h}$ was statistically significant.

miR-21 regulates macrophage apoptosis induced by high levels of glucose. As cell apoptosis has previously been observed to increase with increased miR-21 expression (24), the complex role of miR-21 on high-glucose-induced macrophage apoptosis was investigated. Cells were transfected with 


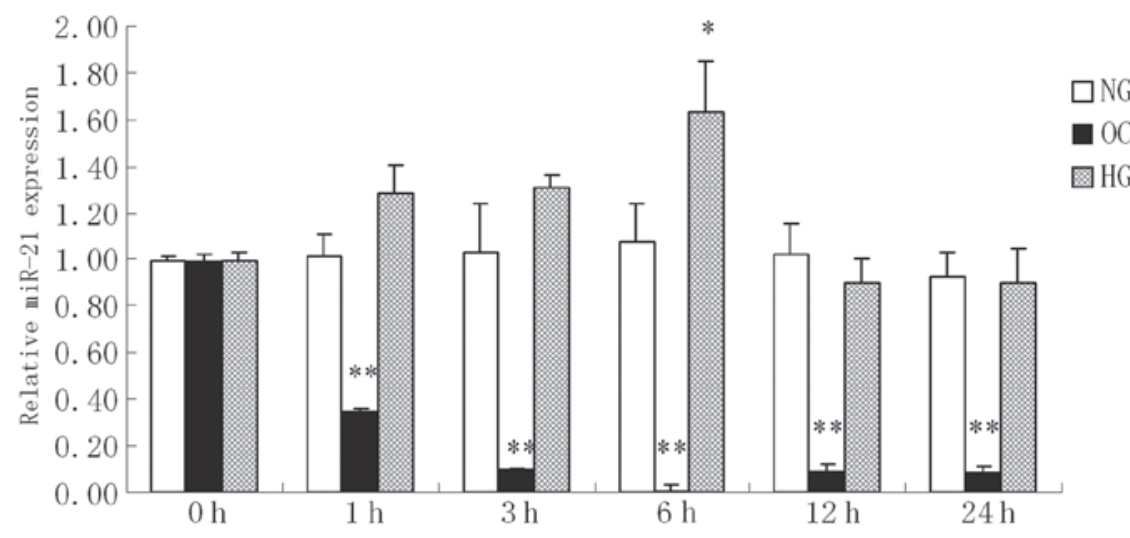

Figure 1. miR-21 expression was upregulated by a high glucose concentration. TaqMan reverse transcription-quantitative polymerase chain reaction analysis of Raw 264.7 macrophages treated with NG (5.5 mM glucose), HG (25 mM glucose) or OC ( $25 \mathrm{mM}$ mannose) for 0, 1, 3, 6, 12 and $24 \mathrm{~h}$. "P<0.05 and "** P<0.01, compared with NG cells. mir-21, microRNA-21; NG, normal glucose; HG, high glucose; OC, osmotic control.

an anti-miR-21 inhibitor or a negative control, and incubated for an additional $48 \mathrm{~h}$ with NG or HG. Apoptosis of cells was detected by flow cytometry. miR-21 mRNA expression was significantly decreased following anti-miR-21 inhibitor transfection (Fig. 2). The results of the flow cytometric analysis demonstrated that apoptosis of macrophages transfected with the anti-miR-21 inhibitor was significantly higher than in those transfected with negative control and incubated with NG (Fig. 3A). In addition, miR-21-inhibited cells treated with HG exhibited a significant increase in apoptosis compared with HG-treated negative control cells, or cells treated with miR-21 inhibition alone (the NG group). The results indicated that miR-21 had a protective effect against high-concentration glucose-induced macrophage apoptosis.

Western blot analysis (Fig. 3B) indicated an increased level of activated caspase-3 protein in miR-21-inhibited cells compared with that in control cells, an increased level with HG-treated miR-21-inhibited cells compared with that in HG-treated controls, and an increased level with the miR-21 inhibitor alone (NG group) compared with that in cells treated with negative controls.

PDCD4 expression in macrophages incubated with high levels of glucose. Raw 264.7 macrophages were treated with $\mathrm{NG}, \mathrm{HG}$ or OC for various time periods and the expression of PDCD4 mRNA was then determined. As shown in Fig. 4A, macrophages treated with $\mathrm{HG}$ revealed a markedly reduced expression of PDCD4 mRNA at $12 \mathrm{~h}$, which was increased from $24 \mathrm{~h}$ compared with NG control cells. However, PDCD4 mRNA expression was significantly increased after $6 \mathrm{~h}$ in the OC group. PDCD4 protein level was also appeared to be reduced following $\mathrm{HG}$ treatment for $12 \mathrm{~h}$ (Fig. 4B).

Inhibition of miR-21 promotes PDCD4 expression. A previous study demonstrated that PDCD4 is a potential miR-21 target in various cell types (22). in order to verify PDCD4 as a target gene of miR-21 in high-glucose-incubated macrophages, the anti-miR-21 inhibitor was transfected into macrophages to inhibit miR-21 expression. Following NG treatment, the mRNA and protein expression of PDCD4 in macrophages transfected with anti-miR-21 inhibitor was higher than in those transfected with negative control (Fig. 5), which indicated that

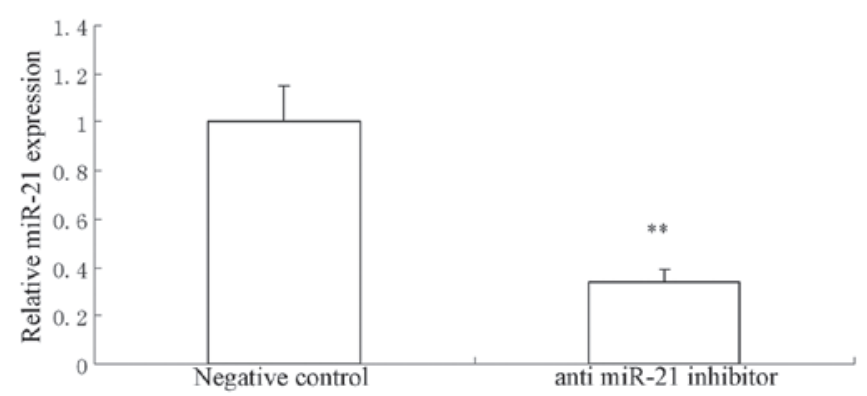

Figure 2. Expression of miR-21 mRNA following transfection with an anti-miR-21 inhibitor. Raw 264.7 macrophages were transfected with an anti-miR-21 inhibitor or negative control for $24 \mathrm{~h}$, and then TaqMan reverse transcription-quantitative polymerase chain reaction was performed to analyze miR-21 mRNA expression. ${ }^{* *} \mathrm{P}<0.01$, compared with negative control. mir-21, microRNA-21.

in macrophages, miR-21 inhibition promotes PDCD4 expression and that PDCD4 is the target gene of miR-21. Following HG treatment, PDCD4 expression in miR-21-inhibited macrophages remained higher than in cells transfected with negative control, but lower than that in miR-21-inhibited cells followed by treatment with NG, which indicates that high concentrations of glucose may reduce PDCD4 expression of macrophages via an miR-21 pathway.

\section{Discussion}

In the present study, it was demonstrated that miR-21 was sensitive to high glucose treatment in Raw 264.7 macrophages. The expression of miR-21 was significantly upregulated at $6 \mathrm{~h}$, and a non-significant time-dependent increase was observed at the other time points in response to high glucose stimulation. In addition, miR-21 is able to resist macrophage apoptosis induced by high glucose via its action on the target gene, PDCD4.

miR-21, located on chromosome 17q23.2, exhibits a clear evolutionary conservation across a number of species and a markedly conserved role in gene regulation (26). miR-21 is reported to be universally expressed in mammalian organ systems, including the heart, spleen, colon and small intestine (27), whereas aberrant expression is observed in 
A

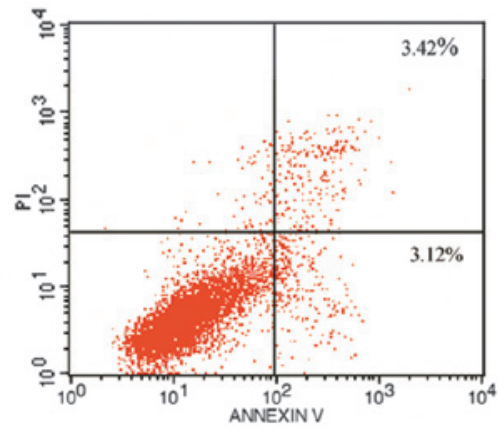

Negative control+NG

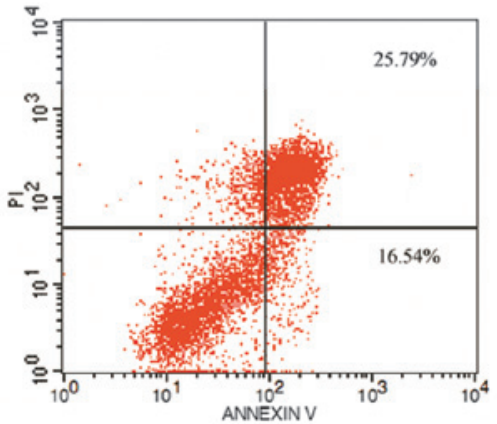

Negative control+HG

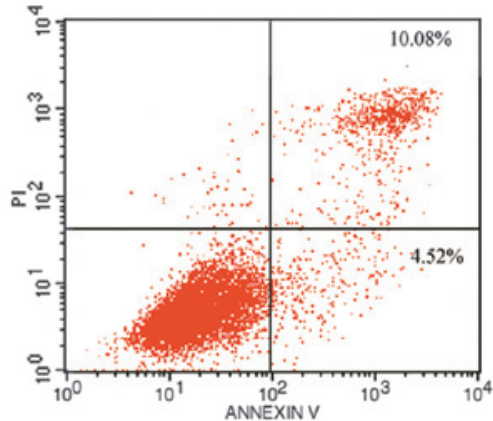

anti-miR-21 inhibitor + NG

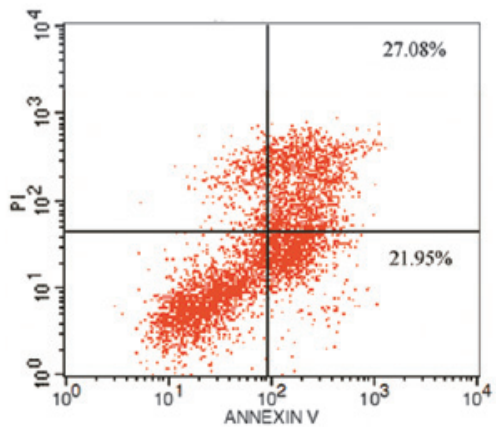

anti-miR-21 inhibitor + HG

B

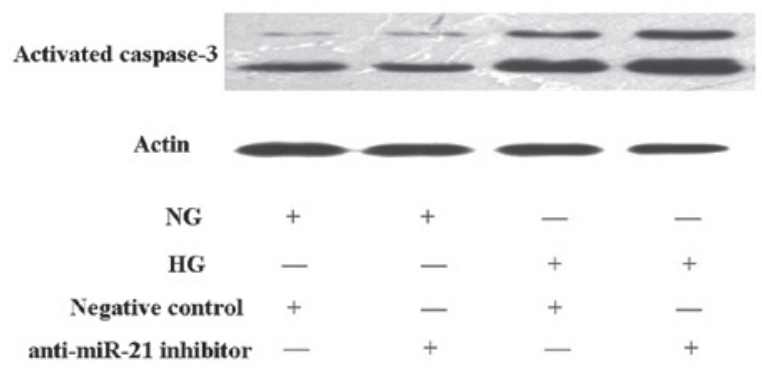

Figure 3. Effect of miR-21 inhibition on macrophage apoptosis. Raw 264.7 macrophages were transfected with an anti-miR-21 inhibitor or negative control for $24 \mathrm{~h}$ prior to incubation for an additional $48 \mathrm{~h}$ with NG $(5.5 \mathrm{mM}$ glucose) or $\mathrm{HG}$ ( $25 \mathrm{mM}$ glucose). (A) Flow cytometry analysis of cell apoptosis by propidium iodide-Annexin V staining. (B) Western blot analysis of expression of activated caspase-3 protein. mir-21, microRNA-21; NG, normal glucose; HG, high glucose.

A

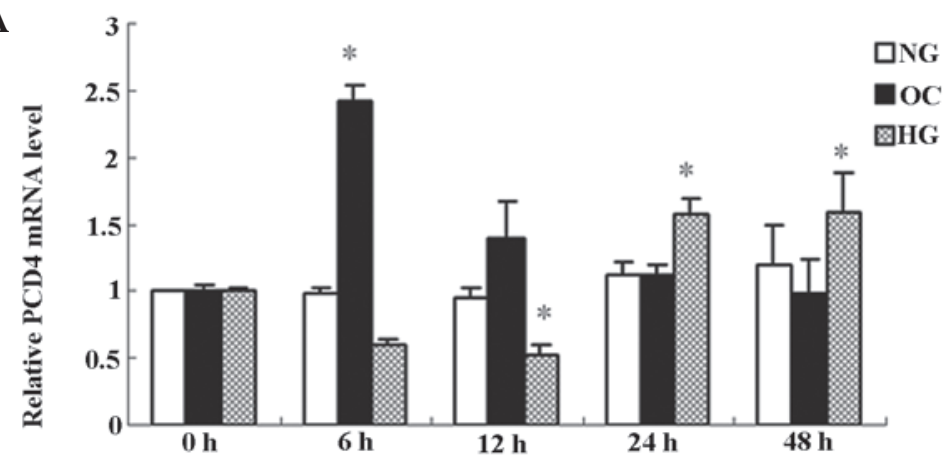

B

Figure 4. PDCD4 expression in macrophages incubated with high glucose. Raw 264.7 macrophages were treated with NG (5.5 mM glucose), HG (25 mM glucose) or OC ( $25 \mathrm{mM}$ mannose) for $0,6,12,24$ and $48 \mathrm{~h}$. (A) Reverse transcription-quantitative polymerase chain reaction analysis of PDCD4 mRNA expression in Raw 264.7 macrophages. " $\mathrm{P}<0.05,{ }^{* *} \mathrm{P}<0.01$, compared with NG cells. (B) Western blot analysis of expression of PDCD4 protein in Raw 264.7 macrophages. NG, normal glucose; HG, high glucose; OC, osmotic control; PDCD4, programmed cell death 4.

atherosclerosis, cardiac hypertrophy, heart failure and acute myocardial infarction (19). miR-21 expression has been revealed to be augmented by multiple stimuli, including lipopolysaccharides, shear stress, hydrogen peroxide, transforming growth factor- $\beta$, angiotensin II and ischemia/reperfusion injury (20,28-31). However, few studies 
A

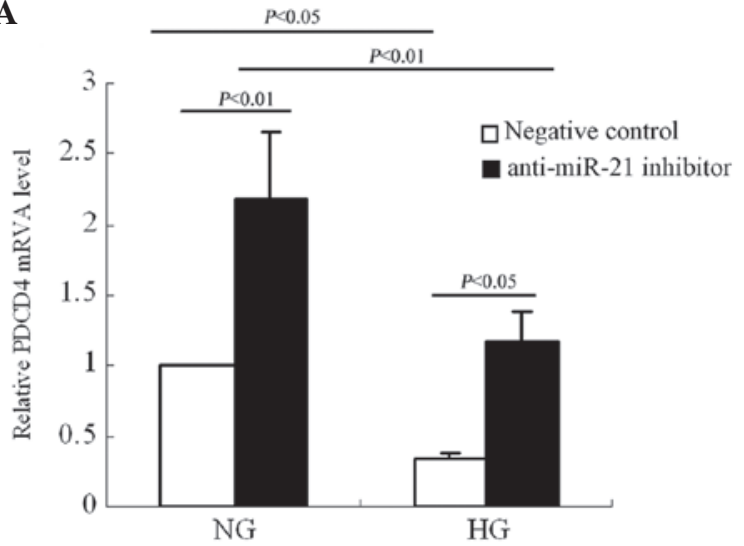

B

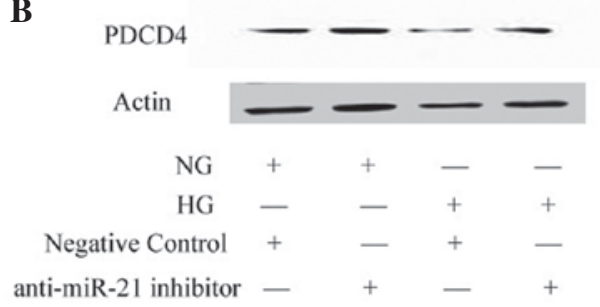

Figure 5. Effect of miR-21 inhibition on PDCD4 expression. Raw 264.7 macrophages were transfected with anti-miR-21 inhibitor or negative control for $24 \mathrm{~h}$ prior to being incubated for an additional $12 \mathrm{~h}$ with $\mathrm{NG}(5.5 \mathrm{mM}$ glucose) or HG (25 mM glucose). (A) Reverse transcription-quantitative polymerase chain reaction analysis of PDCD4 mRNA expression. (B) Western blot analysis of PDCD4 protein expression. NG, normal glucose; HG, high glucose OC, osmotic control; PDCD4, programmed cell death 4.

have been conducted regarding the expression of miR-21 in macrophages. In the present study, it was also observed that miR-21 expression was sensitive to high glucose in Raw 264.7 macrophages and increased following high concentration glucose treatment in a time-dependent manner, which was in accordance with the results of Sheedy et al (28), who found that lipopolysaccharide treatment induced miR-21 expression in Raw 264.7 macrophages, whilst it did not have the same effect in the human acute monocytic leukemia cell line.

miR-21, as a novel gene regulator, promotes cell proliferation and survival, and inhibits cell apoptosis. miR-21 has been observed to inhibit cell apoptosis in mouse periovulatory granulose cells (32). Downregulation of miR-21 expression by antisense oligonucleotides induced cell apoptosis by activating caspase-9 and caspase-3 (33). Furthermore, following treatment with an adenovirus expressing miR-21, ischemia-induced cell apoptosis decreased in the infarcted and border areas of the rat heart following acute myocardial infarction (34). miR-21 has been shown to augment ERK-MAP kinase activity through inhibition of sprouty homologue 1 and to promote ERK-MAP kinase-mediated cell survival in cardiac fibroblasts (35). However, the effect of miR-21 on macrophage apoptosis induced by a high glucose concentration remains to be elucidated. Caspase-3, a key molecule in the classical apoptotic pathway, has an important role in high-concentration glucose-induced apoptosis. A high glucose level induces macrophage apoptosis through activation of caspase-3 (36). Therefore, the expression of caspase- 3 is used as the primary indicator of macrophage apoptosis. In order to clarify the function of miR-21 in macrophage apoptosis, Raw 264.7 macrophages were transfected with an anti-miR-21 inhibitor to inhibit the expression of miR-21 in the present study. It was shown that inhibition of miR-21 in macrophages increased cell apoptosis and augmented activated caspase-3 expression, which suggests a role in increased macrophage apoptosis. Apoptosis was further increased in miR-21-inhibited macrophages treated with high glucose. These results suggest that miR-21 has an antiapoptotic effect on macrophage apoptosis induced by high concentrations of glucose, possibly via the caspase-3 pathway. Although high concentrations of glucose may induce macrophage apoptosis through multiple signaling pathways, miR-21 may be a key regulator of macrophage apoptosis.

PDCD4 has been identified as a gene that is upregulated during apoptosis (37). Recently, PDCD4 was found to be a principal target of miR-21 in numerous types of cells, including colon cancer cells, human glioblastoma cells, cardiomyocytes, HUVECs and human peripheral blood mononuclear cells $(19,38-40)$. It remains to be elucidated whether PDCD4 is also a target gene of miR-21 in Raw 264.7 macrophages incubated with high glucose. In order to confirm this, Raw 264.7 macrophages were treated with a high concentration of glucose for various time periods, demonstrating that mRNA and protein levels of PDCD4 were downregulated by a high concentration of glucose, which suggested PDCD4 is a potential target of miR-21.

To further clarify the interaction between miR-21 and PDCD4 in high-concentration glucose-treated Raw 264.7 macrophages, miR-21 expression was modulated via the use of an anti-miR-21 inhibitor and the change in PDCD4 expression was observed. It was identified that PDCD4 expression was significantly increased following inhibition of miR-21 expression, which demonstrated that miR-21 suppresses PDCD4 expression. In addition, treatment of macrophages with an miR-21 inhibitor and a high concentration of glucose, markedly increased PDCD4 expression compared with that in cells treated with an miR-21 inhibitor and normal glucose. Previous studies have demonstrated that miR-21 binds to the 3'-untranslated region (UTR) of the PDCD4 gene at nucleotides 228-249 (41). Based upon this finding, it is hypothesized that PDCD4 is directly suppressed by miR-21 in macrophages and is involved in macrophage apoptosis induced by high concentrations of glucose. In the early stages of macrophage apoptosis, a high level of glucose primarily induces the expression of miR-21, which suppresses PDCD4 expression by binding to the 3'-UTR of PDCD4. In addition, the induction of miR-21 resists high-concentration glucose-independent macrophage apoptosis. However, in the late stages of apoptosis, high glucose levels cause a high level of cell apoptosis, accompanied by markedly increased PDCD4 expression,which suggests that upregulated PDCD4 may regulate miR-21 expression induced by high concentrations of glucose in a negative feedback manner.

Macrophage apoptosis has been observed to promote vulnerable atherosclerotic plaque progression. Since it has been identified that miR-21 is implicated in reducing macrophage apoptosis that is induced by high concentrations of glucose, and that miR-21 may inhibit high-concentration glucose-induced apoptosis, miR-21 may have a protective role in the development of vulnerable atherosclerotic plaques. 
However, further studies are required in order to elucidate the underlying mechanisms. The preliminary findings from the present study suggest that miR-21 may be a novel therapeutic target in atherosclerosis.

In conclusion, a high concentration of glucose induces the expression of miR-21 in Raw 264.7 macrophages. miR-21 appears to have a protective effect against macrophage apoptosis induced by high concentrations of glucose via PDCD4. An investigation of the association between mannose and gene-mediated apoptosis may be beneficial in the future in order to expand this field of knowledge.

\section{Acknowledgements}

The present study was supported by research grants from the National Basic Research Program of China (973 program; grant no. 2012CB722406), the National Natural Science Foundation of China (grant nos. 30971215, 81070192,81070141, 81100605, 81270352 and 81270287), the Natural Science Foundation of Shandong Province (grant nos. BS2011YY013 and BS2013YY017), the Independent Innovation Foundation of Shandong University (grant no. 2012JC034) and the CardiovascularExploration Research Foundation of the Chinese Medical Doctors Association (grant no. DFCMDA201320).

\section{References}

1. Bornfeldt KE and Tabas I: Insulin resistance, hyperglycemia and atherosclerosis. Cell Metab 14: 575-585, 2011.

2. Hurst RT and Lee RW: Increased incidence of coronary atherosclerosis in type 2 diabetes mellitus: mechanisms and management. Ann Intern Med 139: 824-834, 2003.

3. Tabas I: Consequences and therapeutic implications of macrophage apoptosis in atherosclerosis: the importance of lesion stage and phagocytic efficiency. Arterioscler Thromb Vasc Biol 25: 2255-2264, 2005.

4. Kolodgie FD, Narula J, Burke AP, Haider N, Farb A, Hui-Liang Y, Smialek J and Virmani R: Localization of apoptotic macrophages at the site of plaque rupture in sudden coronary death. Am J Pathol 157: 1259-1268, 2000.

5. Liang CP, Han S, Senokuchi T and Tall AR: The macrophage at the crossroads of insulin resistance and atherosclerosis. Circ Res 100: 1546-1555, 2007.

6. Burke AP, Kolodgie FD, Zieske A, Fowler DR, Weber DK, Varghese PJ, Farb A and Virmani R: Morphologic findings of coronary atherosclerotic plaques in diabetics: a postmortem study. Arterioscler Thromb Vasc Biol 24: 1266-1271, 2004.

7. Griffiths-Jones S, Grocock RJ, van Dongen S, Bateman A and Enright AJ: miRBase: microRNA sequences, targets and gene nomenclature. Nucleic Acids Res 34: D140-D144, 2006.

8. Zamore PD and Haley B: Ribo-gnome: the big world of small RNAs. Science 309: 1519-1524, 2005.

9. Bentwich I, Avniel A, Karov Y, Aharonov R, Gilad S, Barad O, Barzilai A, Einat P, Einav U, Meiri E, et al: Identification of hundreds of conserved and nonconserved human microRNAs. Nat Genet 37: 766-770, 2005.

10. Lewis BP, Burge CB and Bartel DP: Conserved seed pairing, often flanked by adenosines, indicates that thousands of human genes are microRNA targets. Cell 120: 15-20, 2005.

11. Bartel DP: MicroRNAs: genomics, biogenesis, mechanism and function. Cell 116: 281-297, 2004.

12. Nugent M, Miller N and Kerin MJ: MicroRNAs in colorectal cancer: function, dysregulation and potential as novel biomarkers. Eur J Surg Oncol 7: 649-654, 2011.

13. Hennessy E, Clynes M, Jeppesen PB and O'Driscoll L: Identification of microRNAs with a role in glucose stimulated insulin secretion by expression profiling of MIN6 cells. Biochem Biophys Res Commun 396: 457-462, 2010.

14. Urbich C, Kuehbacher A and Dimmeler S: Role of microRNAs in vascular diseases, inflammation, and angiogenesis. Cardiovasc Res 79: 581-588, 2008.
15. Han M, Toli J and Abdellatif M: MicroRNAs in the cardiovascular system. Curr Opin Cardiol 26: 181-189, 2011.

16. Folini M, Gandellini P,Longoni N, Profumo V, Callari M,Pennati M, Colecchia M, Supino R, Veneroni S, Salvioni R, et al: miR-21: an oncomir on strike in prostate cancer. Mol Cancer 9: 12, 2010.

17. Cheng Y, Ji R, Yue J, Yang J, Liu X, Chen H, Dean DB and Zhang C: MicroRNAs are aberrantly expressed in hypertrophic heart: do they play a role in cardiac hypertrophy? Am J Pathol 170: 1831-1840, 2007

18. Roy S, Khanna S, Hussain SR, Biswas S, Azad A, Rink C, Gnyawali S, Shilo S, Nuovo GJ and Sen CK: MicroRNA expression in response to murine myocardial infarction: miR-21 regulates fibroblast metalloprotease- 2 via phosphatase and tensin homologue. Cardiovasc Res 82: 21-29, 2009.

19. Cheng Y and Zhang C: MicroRNA-21 in cardiovascular disease. J Cardiovasc Transl Res 3: 251-255, 2010.

20. Weber M, Baker MB, Moore JP and Searles CD: MiR-21 is induced in endothelial cells by shear stress and modulates apoptosis and eNOS activity. Biochem Biophys Res Commun 393: 643-648, 2010.

21. Ji R, Cheng Y, Yue J, Yang J, Liu X, Chen H, Dean DB and Zhang C: MicroRNA expression signature and antisense-mediated depletion reveal an essential role of MicroRNA in vascular neointimal lesion formation. Circ Res 100: 1579-1588, 2007.

22. Raitoharju E, Lyytikäinen LP, Levula M, Oksala N, Mennander A, Tarkka M, Klopp N, Illig T, Kähönen M, Karhunen PJ, et al: miR-21, miR-210, miR-34a and miR-146a/b are up-regulated in human atherosclerotic plaques in the Tampere vascular study. Atherosclerosis 219: 211-217, 2011.

23. Davis BN, Hilyard AC, Lagna G and Hata A: SMAD proteins control DROSHA-mediated microRNA maturation. Nature 454: 56-61, 2008.

24. Cheng Y, Liu X, Zhang S, Lin Y, Yang J and Zhang C: MicroRNA-21 protects against the $\mathrm{H}(2) \mathrm{O}(2)$-induced injury on cardiac myocytes via its target gene PDCD4. J Mol Cell Cardiol 47: 5-14, 2009.

25. Livak KJ and Schmittgen TD: Analysis of relative gene expression data using real-time quantitative PCR and the $2^{-\Delta \Delta C T}$ method. Methods 25: 402-408, 2001.

26. Selcuklu SD, Donoghue MT and Spillane C: miR-21 as a key regulator of oncogenic processes. Biochem Soc Trans 37: 918-925, 2009.

27. Lagos-Quintana M, Rauhut R, Yalcin A, Meyer J, Lendeckel W and Tuschl T: Identification of tissue-specific microRNAs from mouse. Curr Biol 12: 735-739, 2002.

28. Sheedy FJ, Palsson-McDermott E, Hennessy EJ, Martin C, O'Leary JJ, Ruan Q, Johnson DS, Chen Y and O'Neill LA: Negative regulation of TLR4 via targeting of the proinflammatory tumor suppressor PDCD4 by the microRNA miR-21. Nat Immunol 11: 141-147, 2010.

29. Lin Y, Liu X, Cheng Y, Yang J, Huo Y and Zhang C: Involvement of MicroRNAs in hydrogen peroxide-mediated gene regulation and cellular injury response in vascular smooth muscle cells. J Biol Chem 284: 7903-7913, 2009.

30. Yin C, Wang $\mathrm{X}$ and Kukreja RC: Endogenous microRNAs induced by heat-shock reduce myocardial infarction following ischemia-reperfusion in mice. FEBS Lett 582: 4137-4142, 2008.

31. Tatsuguchi M, Seok HY, Callis TE, Thomson JM, Chen JF, Newman M, Rojas M, Hammond SM and Wang DZ: Expression of microRNAs is dynamically regulated during cardiomyocyte hypertrophy. J Mol Cell Cardiol 42: 1137-1141, 2007.

32. Carletti MZ, Fiedler SD and Christenson LK: MicroRNA 21 blocks apoptosis in mouse periovulatory granulosa cells. Biol Reprod 83: 286-295, 2010.

33. Zhou X, Zhang J, Jia Q, Ren Y, Wang Y, Shi L, Liu N, Wang G, $\mathrm{Pu}$ P, You Y and Kang C: Reduction of miR-21 induces glioma cell apoptosis via activating caspase 9 and 3. Oncol Rep 24: 195-201, 2010.

34. Dong S, Cheng Y, Yang J, Li J, Liu X, Wang X, Wang D, Krall TJ, Delphin ES and Zhang C: MicroRNA expression signature and the role of microRNA-21 in the early phase of acute myocardial infarction. J Biol Chem 284: 29514-29525, 2009.

35. Thum T, Gross C, Fiedler J, Fischer T, Kissler S, Bussen M, Galuppo P, Just S, Rottbauer W, Frantz S, et al: MicroRNA-21 contributes to myocardial disease by stimulating MAP kinase signalling in fibroblasts. Nature 456: 980-984, 2008.

36. Liang JL, Xiao DZ, Liu XY, Lin QX, Shan ZX, Zhu JN, Lin SG and Yu XY: High glucose induces apoptosis in AC16 human cardiomyocytes via macrophage migration inhibitory factor and c-Jun N-terminal kinase. Clin Exp Pharmacol Physiol 37: 969-973, 2010. 
37. Liu X, Cheng Y, Yang J, Krall TJ, Huo Y and Zhang C: An essential role of PDCD4 in vascular smooth muscle cell apoptosis and proliferation: implications for vascular disease. Am J Physiol Cell Physiol 298: C1481-C1488, 2010.

38. Yu Y, Kanwar SS, Patel BB, Oh PS, Nautiyal J, Sarkar FH and Majumdar AP: MicroRNA-21 induces stemness by downregulating transforming growth factor beta receptor 2 (TGF $\beta R 2$ ) in colon cancer cells. Carcinogenesis 33: 68-76, 2012.

39. Gaur AB, Holbeck SL, Colburn NH and Israel MA: Downregulation of Pdcd4 by mir-21 facilitates glioblastoma proliferation in vivo. Neuro Oncol 13: 580-590, 2011.
40. Cheng Y, Zhu P, Yang J, Liu X, Dong S, Wang X, Chun B, Zhuang $J$ and Zhang C: Ischaemic preconditioning-regulated miR-21 protects heart against ischaemia/reperfusion injury via anti-apoptosis through its target PDCD4. Cardiovasc Res 87: 431-439, 2010.

41. Allgayer H: Pdcd4, a colon cancer prognostic that is regulated by a microRNA. Crit Rev Oncol Hematol 73: 185-191, 2010. 